\title{
Mangiferin ameliorates cardiac fibrosis in D-galactose-induced aging rats by inhibiting TGF- $\beta /$ p38/MK2 signaling pathway
}

\author{
Jing Cheng ${ }^{1}$, Chaoyang Ren², Renli Cheng ${ }^{3}$, Yunning Li ${ }^{4}$, Ping Liu' ${ }^{1}$, Wei Wang ${ }^{1, *}$, and Li Liu ${ }^{1, *}$ \\ ${ }^{1}$ Department of Pharmacy, Wuhan Fourth Hospital, Puai Hospital, Tongji Medical College, Huazhong University of Science and Technology, ${ }^{2}$ Dong Xi Hu Mu- \\ nicipal Healthcare Security Administration, ${ }^{3}$ Department of Orthopedics, Wuhan Fourth Hospital, Puai Hospital, Tongji Medical College, Huazhong University \\ of Science and Technology, Wuhan 430033, Hubei, ${ }^{4}$ Department of Traditional Chinese Medicine, 986 Hospital of Air Force, Xian 710054, Shanxi, China
}

\section{ARTICLE INFO}

Received August 25, 2020

Revised October 24, 2020

Accepted November 3, 2020

\section{*Correspondence}

Li Liu

E-mail: puaichengjing@163.com

Wei Wang

E-mail: doudoumav@sina.com

Key Words

Aging

Heart fibrosis

Inflammation

Mangiferin

Oxidative stress

\begin{abstract}
Aging is the process spontaneously occurred in living organisms. Cardiac fibrosis is a pathophysiological process of cardiac aging. Mangiferin is a wellknown C-glucoside xanthone in mango leaves with lots of beneficial properties. In this study, rat model of cardiac fibrosis was induced by injected with $150 \mathrm{mg} / \mathrm{kg} / \mathrm{d}$ Dgalactose for 8 weeks. The age-related cardiac decline was estimated by detecting the relative weight of heart, the serum levels of cardiac injury indicators and the expression of hypertrophic biomakers. Cardiac oxidative stress and local inflammation were measured by detecting the levels of malondialdehyde, enzymatic antioxidant status and proinflammatory cytokines. Cardiac fibrosis was evaluated by observing collagen deposition via masson and sirius red staining, as well as by examining the expression of extracellular matrix proteins via Western blot analysis. The cardiac activity of profibrotic TGF- $\beta 1 / p 38 /$ MK2 signaling pathway was assessed by measuring the expression of TGF- $\beta 1$ and the phosphorylation levels of p38 and MK2. It was observed that mangiferin ameliorated D-galactose-induced cardiac aging, attenuated cardiac oxidative stress, inflammation and fibrosis, as well as inhibited the activation of TGF- $\beta 1 / p 38 / M K 2$ signaling pathway. These results showed that mangiferin could ameliorate cardiac fibrosis in D-galactose-induced aging rats possibly via inhibiting TGF- $\beta / p 38 /$ MK2 signaling pathway.
\end{abstract}

\section{INTRODUCTION}

Population aging is a global public health problem [1]. Aging is the deteriorative process spontaneously occurred in living organisms and characterized by progressive decline in multiple organ functions and systemic immunity [2]. As a result, aging is suggested to be a major risk factor for varieties of diseases. It is reported that cardiovascular diseases (CVDs) (including hypertension, cardiac hypertrophy and heart failure) contribute to about $31 \%$ of all deaths worldwide [3]. In fact, CVDs have already been the major health problems in both developed countries and developing countries [4]. Aging affects the heart at functional, structural and molecular levels $[5,6]$. These aging-related CVDs have become the leading cause of death in elderly people [1]. CVDs, especially cardiac hypertrophy, are the common pathological changes in aging process [7]. Cardiac hypertrophy is a compensatory change of the heart in order to adapt to hypertension, vascular heart diseases and so on [7]. Cardiac hypertrophy and its accompanied myocardial fibrosis are usually responsible for heart failure [4]. Extracellular matrix (ECM) remodeling and collagen deposition are the characteristics of organ fibrosis. Aging promotes cardiac fibrosis [8]. However, there is no effective treatment for attenuating the aging-associated cardiac hypertrophy and fibrosis [9].

Mangiferin is a well-known C-glucoside xanthone and widespread in herbal medicines and fruits including mango leaves (Mangifera indica Linn) [10]. Mangiferin is known to has varies

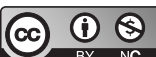

This is an Open Access article distributed under the terms of the Creative Commons Attribution Non-Commercial License, which permits unrestricted non-commercial use, distribution, and reproduction in any medium, provided the original work is properly cited. Copyright @ Korean J Physiol Pharmacol, pISSN 1226-4512, elSSN 2093-3827
Author contributions: J.C., W.W., and L.L. performed the conception and design of study. J.C. wrote the manuscript and performed the Western blot analysis. C.R. and R.C. conducted the acquisition analysis and interpretation of data. Y.L. and P.L. conducted part of the experiments and revised the manuscript. 
of beneficial properties including anti-apoptotic, anti-oxidative and anti-inflammatory [11]. Reports showed that mangiferin administration ameliorated isoproterenol-induced apoptosis [12] and hydrogen peroxide-induced cell injury [10] in cardiomyocytes. Mangiferin was also believed to can protect against myocardial ischemia-reperfusion injury [11]. Furthermore, mangiferin has the functions of attenuating transverse aortic constrictioninduced cardiac fibrosis [13]. Therefore, the potential protective effects of mangiferin on heart attract a great deal of attentions. This study was aimed to investigate the potential ameliorative effects of mangiferin against cardiac fibrosis in D-galactose (D-gal)induced aging rats and its underlying molecular mechanisms.

\section{METHODS}

\section{Chemicals and reagents}

Mangiferin (purity $>95 \%$ ) and D-gal (purity $>99 \%$ ) were purchased from Aladdin Co, Ltd. (Shanghai, China). MMI-0100 (sequence: YARAAARQARAKALARQLGVAA) was synthesized by Genscript Biotechnology, Co. (Jiangsu, China). The commercial kits for the analysis of creatine kinase (CK), lactate dehydrogenase (LDH), superoxide dismutase (SOD), catalase (CAT) and malondialdehyde (MDA) were purchased from Nanjing Jiancheng Bioengineering Institute (Jiangsu, China). The commercial kits for the analysis of interleukin (IL)-1 $\beta$ IL- 6 and tumor necrosis factor (TNF)- $\alpha$ were purchased from ELISA Lab, Co. Ltd. (Hubei, China). The antibodies of phosphorylation-mitogen activated protein kinase activated protein kinase II (p-MK2) and $\beta$-myosin heavy chain ( $\beta$-MHC) were purchased from Santa Cruz Biotechnology, Inc. (Santa Cruz, CA, USA). The antibodies of p-p38, $\alpha$-smooth muscle actin ( $\alpha$-SMA), collagen (Col)-I, ColIII, natriuretic peptides A (ANP) and BNP were purchased from from Abcam, Co. (Cambridge, UK). The antibody of transforming growth factor- $\beta 1$ (TGF- $\beta 1$ ) was purchased from Proteintech Group, Inc. (Chicago, IL, USA).

\section{Animals and groups}

Eight weeks adult male Wistar rats (180-220 g) were obtained from the Centers of Disease Control and Prevention of Hubei Province, China. Rats were maintained under standard environment (temperature of $22^{\circ} \mathrm{C} \pm 3^{\circ} \mathrm{C}$, humidity of $50 \% \pm 10 \%$ and $12 \mathrm{~h}$ light:12 $\mathrm{h}$ dark cycle). After acclimatization to the laboratory conditions for one week, the rats were randomly assigned into 5 groups $(\mathrm{n}=8)$ : the vehicle control group (Veh), the D-gal-induced aging group (aging), the aging-MMI-0100 group (MMI-0100), the aging- $100 \mathrm{mg} / \mathrm{kg}$ mangiferin group (100 mangiferin) and aging-50 $\mathrm{mg} / \mathrm{kg}$ mangiferin group (50 mangiferin). The rats from aging, MMI-0100, 100 mangiferin and 50 mangiferin groups were injected with $150 \mathrm{mg} / \mathrm{kg} / \mathrm{d} \mathrm{D}$-gal for 8 weeks [14]. Animals from 100 mangiferin and 50 mangiferin groups were daily intragastric administration of 100 and $50 \mathrm{mg} / \mathrm{kg}$ mangiferin for 8 weeks, respectively. The low dose of mangiferin was set according to previous report [13]. The high dose of mangiferin was set as 2 times of low dose. The rats from MMI-0100 were daily intraperitoneal injected with $50 \mu \mathrm{g} / \mathrm{kg}$ MMI-0100 [15]. At the end of the 8 weeks experimental period, the animals were anesthetized with pentobarbital $\mathrm{Na}$ (i.p. $100 \mathrm{mg} / \mathrm{kg}$ ). Heart tissue samples were collected, weighted and excised. One part fresh tissue was used for subsequent Western blot analysis. One part tissue was fixed in $4 \%$ paraformaldehyde, embedded in paraffin and cut into thin sections. Then, the sections were stained by masson and sirius red. One part tissue was made to the $10 \%$ tissue homogenate using ice cold $0.9 \%$ sodium chloride for the subsequent assay of cardiac oxidative stress and inflammation. All the experiments were performed in accordance with the Chinese legislation and the ethical rules of NIH Guidelines for the Care and Use of Laboratory Animal, and were approved by the ethics committee of affiliated Puai Hospital of Tongji Medical College, Huazhong University of Science and Technology (approval No. PAH2018-12).

\section{Cardiac aging and fibrosis evaluation}

The age-related cardiac decline was estimated by detecting the relative weight of heart, the levels of serum cardiac markers (CK and $\mathrm{LDH}$ ) and the expression of hypertrophic makers (ANP, BNP, and $\beta$-MHC) [16]. The relative weight of heart was showed as heart index (HI). HI (mg/g) = the weight of heart (mg) / the weight of body (g). The levels of serum CK and LDH were detected using commercial kits according to instructions. The expression of cardiac ANP, BNP, and $\beta$-MHC was detected by Western blot analysis. Rat heart tissue was ground in liquid nitrogen. The protein samples were extracted and separated by $10 \%$ SDS-polyacrylamide gel electrophoresis. Then, the samples were transferred to a PVDF membrane via electrophoretic transferring and blocked for $1 \mathrm{~h}$ using $5 \%$ nonfat milk in Tris-buffered saline with $0.1 \%$ Tween 20 (TBST). Subsequently, the membranes were incubated with the primary antibody (ANP, BNP, and $\beta-\mathrm{MHC}$ ) overnight at $4^{\circ} \mathrm{C}$, washed with TBST and incubated with horseradish peroxidaseconjugated secondary antibodies in TBST with $3 \%$ nonfat milk for $0.5 \mathrm{~h}$. The chemiluminescence reaction was developed and the quantification of bands was determined by integrated optical density analysis using Alpha Innotech software. The data were normalized using GAPDH as an internal control.

Cardiac fibrosis was evaluated by observing collagen deposition via masson and sirius red staining, as well as by examining the expression of ECM proteins (Col-I, Col-III, and $\alpha$-SMA) via Western blot analysis. The proportion of masson- and sirius redpositive area in sections were measured using Image-Pro Plus 6.0. The mean percent area of positive stained was calculated for 10 randomly selected fields of each section. 


\section{Cardiac oxidative stress and inflammation evaluation}

Rat cardiac oxidative stress was assessed by detecting the activities of enzymatic antioxidants SOD and CAT, as well as the level of lipid peroxidation marker MDA. Cardiac inflammation was evaluated by detecting the levels of IL-1 $\beta$, IL- 6 , and TNF- $\alpha$. The performance was carried out in accordance with the instructions of commercial kits.

\section{Cardiac TGF- $\beta /$ p38/MK2 signaling pathway evaluation}

The cardiac activity of profibrotic TGF- $\beta 1 / \mathrm{p} 38 / \mathrm{MK} 2$ signaling pathway was assessed by measuring the expression of TGF- $\beta 1$ and the phosphorylation levels of p38 and MK2 via Western blot analysis. The data were normalized using GAPDH as an internal control.

\section{Statistical analysis}

The values were presented as mean \pm standard deviation. The bar graphs were made using Origin 6.0. Results were analyzed statistically by one-way ANOVA followed by Tukey's multiple comparison using SPSS 17.0.1 (SPSS Inc., Chicago, IL, USA). Differences were considered as significant at $\mathrm{p}<0.05$.

\section{RESULTS}

\section{Mangiferin ameliorated D-gal-induced cardiac aging and fibrosis in rats}

It can be seen from Table 1 that D-gal injection significantly enhanced the heart weight (wt) and HI compared to the Veh. When compared to the aging group, MMI-0100 and mangiferin (50 and $100 \mathrm{mg} / \mathrm{kg} / \mathrm{d}$ ) significantly reduced the heart wt and HI. As showed in Fig. 1, when compared to the Veh, the serum levels (Fig. 1A) of CK and LDH, as well as the cardiac expression (Fig. 1B) of hypertrophic makers ANP, BNP, and $\beta$-MHC were significantly increased in the rats from Aging group. When compared to the Aging group, MMI-0100 and mangiferin (50 and $100 \mathrm{mg} /$ $\mathrm{kg} / \mathrm{d}$ ) significantly decreased the levels of $\mathrm{CK}$ and $\mathrm{LDH}$, and the expression of ANP and BNP. MMI-0100 and $100 \mathrm{mg} / \mathrm{kg} / \mathrm{d}$ man-

Table 1. Effects of mangiferin on body weight (wt), heart wt and heart index (HI) in D-gal-induced aging rats

\begin{tabular}{|c|c|c|c|}
\hline & Body wt (g) & Heart wt (g) & $\mathrm{HI}(\mathrm{mg} / \mathrm{g})$ \\
\hline Veh & $449.1 \pm 12.5$ & $1.35 \pm 0.07^{* *}$ & $3.00 \pm 0.07^{* *}$ \\
\hline Aging & $454.4 \pm 15.6$ & $1.64 \pm 0.07^{\# \#}$ & $3.61 \pm 0.04^{\# \#}$ \\
\hline MMI-0100 & $452.1 \pm 12.0$ & $1.40 \pm 0.06^{* *}$ & $3.09 \pm 0.06^{* *, \# \#}$ \\
\hline 50 mangiferin & $451.8 \pm 13.8$ & $1.54 \pm 0.07^{*, \# \#}$ & $3.40 \pm 0.07^{* *, * \#}$ \\
\hline 100 mangiferin & $449.9 \pm 13.1$ & $1.47 \pm 0.08^{* *, \#}$ & $3.27 \pm 0.08^{* *, \# \#}$ \\
\hline
\end{tabular}

D-gal, D-galactose; Veh group, vehicle control group; Aging group, D-gal-induced aging group. Compared to aging group, *p $<0.05$, ${ }^{* *} \mathrm{p}<0.01$. Compared to Veh group, ${ }^{\sharp} \mathrm{p}<0.05,{ }^{*} \mathrm{p}<0.01$.
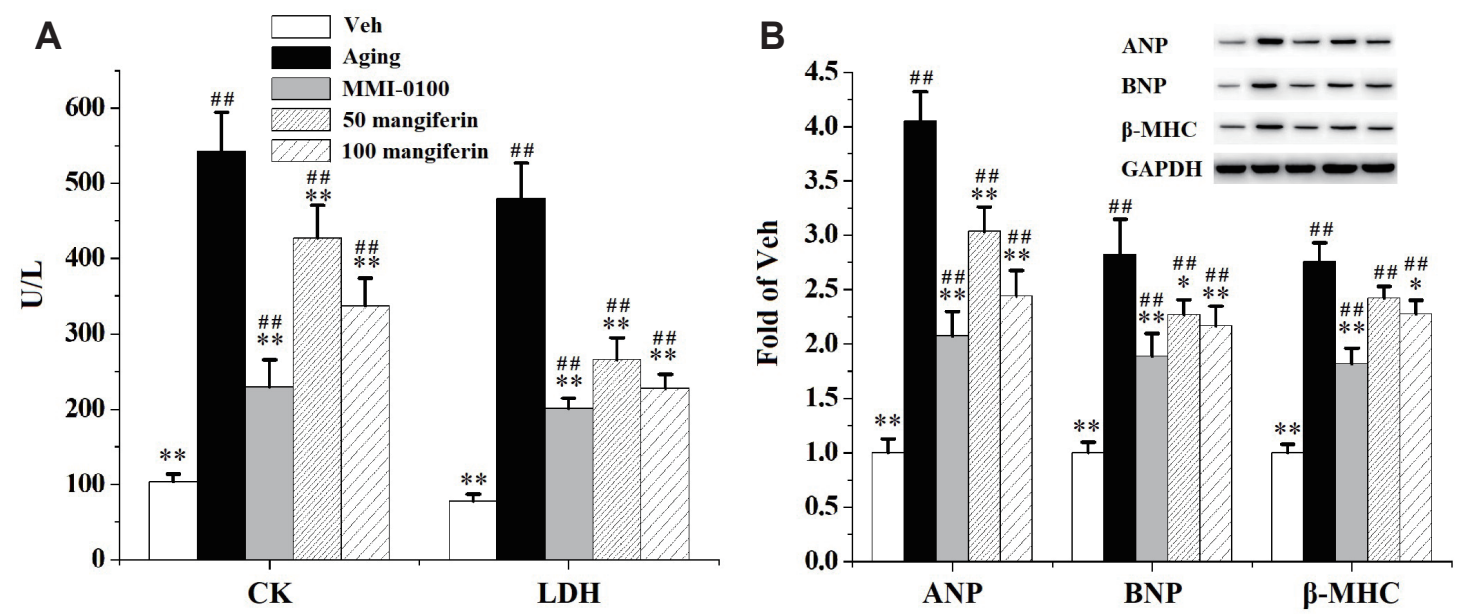

Fig. 1. Effects of mangiferin on serum levels (A) of CK and LDH, as well as on cardiac expression (B) of ANP, BNP, and $\beta$-MHC in D-gal-induced aging rats. The levels of serum CK and LDH were detected using commercial kits according to instructions $(n=8)$. The expression of cardiac ANP, $\mathrm{BNP}$, and $\beta-\mathrm{MHC}$ was detected by Western blot analysis $(\mathrm{n}=3)$. Values were presented as mean \pm standard deviation. $\mathrm{CK}$, creatine kinase; $\mathrm{LDH}$, lactate dehydrogenase; ANP, natriuretic peptides A; $\beta$-MHC, $\beta$-myosin heavy chain; D-gal, D-galactose; Veh group, vehicle control group; Aging group, D-galinduced aging group. ${ }^{*} p<0.05,{ }^{* *} p<0.01$ compared to the Aging group. ${ }^{\# \#} p<0.01$ compared to the Veh group. 
giferin significantly decreased the expression of $\beta$-MHC.

Fig. 2 describes the cardiac collagen deposition. When compared to the Veh, D-gal injection significantly enhanced both the proportion of masson-positive and sirius red-positive area. When compared to the Aging group, MMI-0100 and mangiferin (50 and $100 \mathrm{mg} / \mathrm{kg} / \mathrm{d}$ ) significantly reduced the proportion of massonpositive and sirius red-positive area.

\section{Mangiferin attenuated D-gal-induced cardiac oxidative stress and inflammation in rats}

Fig. 3 describes that D-gal injection stimulates cardiac oxidative stress (Fig. 3A) and inflammation (Fig. 3B). When compared to the Veh, the cardiac activities of SOD and CAT in rats from Aging group were significantly decreased. Meanwhile, the levels of cardiac MDA, IL-1 $\beta$, IL- 6 , and TNF- $\alpha$ were significantly increased in rats from Aging group compared to the Veh. When compared to the Aging group, MMI-0100 and mangiferin (50

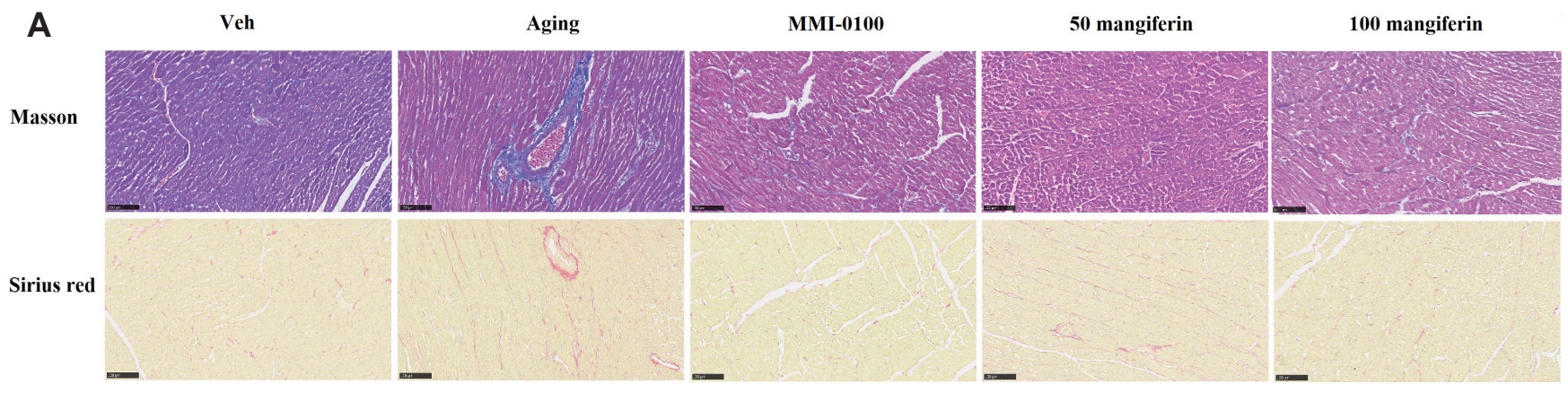

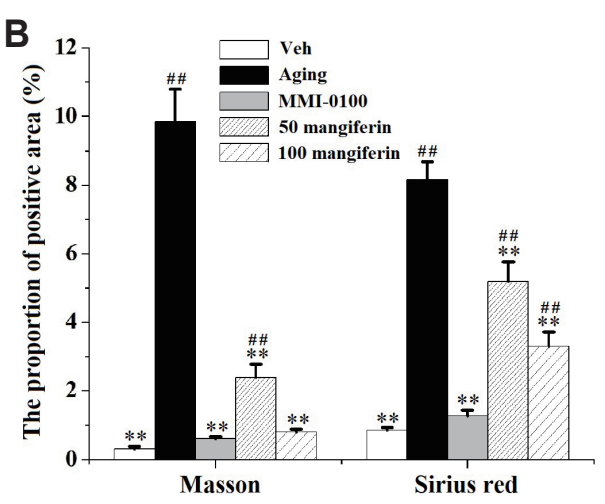

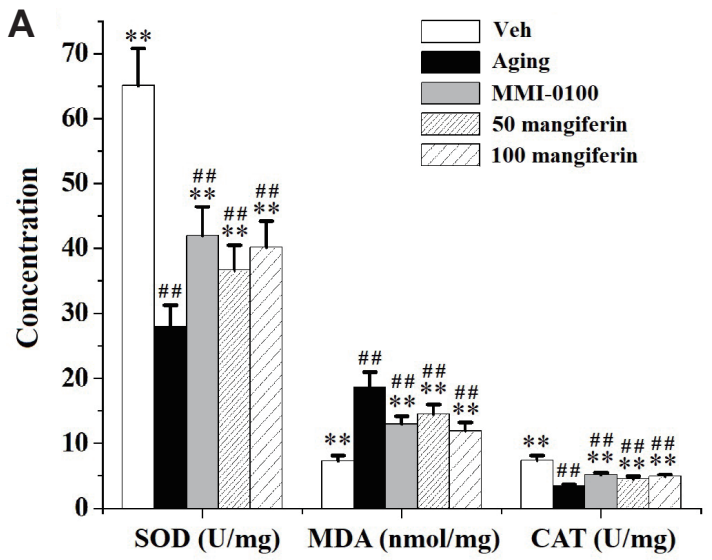

Fig. 2. Effect of mangiferin on cardiac collagen deposition. (A) Histological analysis for the effects of mangiferin on D-gal-induced collagen deposition in rat heart sections stained by masson and sirius red. The proportion of masson- and sirius red-positive area in sections were measured using Image-Pro Plus 6.0. Images were captured at 200x magnification. Scale bar of $100 \mu \mathrm{m}$ was marked. (B) The proportion of masson-positive area and sirius red in heart sections. Values $(n=8)$ were presented as mean \pm standard deviation. D-gal, D-galactose; Veh group, vehicle control group; Aging group, D-gal-induced aging group. ${ }^{* *} \mathrm{p}<0.01$ compared to the Aging group. ${ }^{\# \#} \mathrm{p}<0.01$ compared to the Veh group.

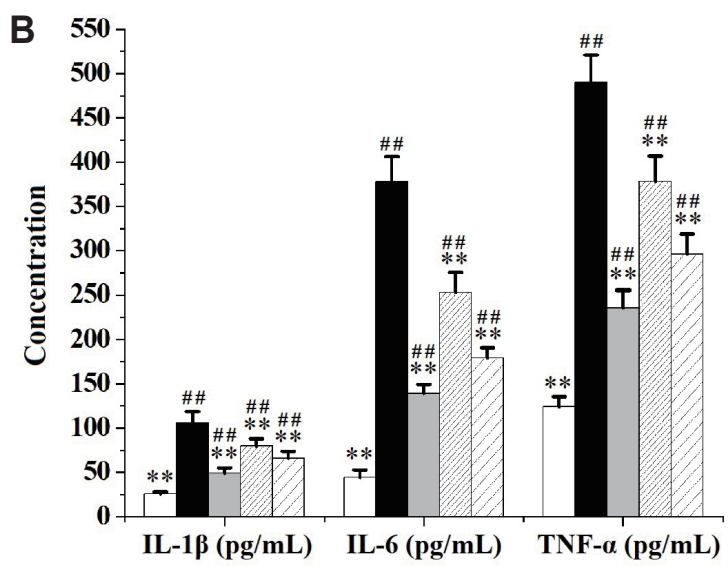

Fig. 3. Effects of mangiferin on cardiac oxidative stress (A) and inflammation (B) in D-gal-induced aging rats. The activities of enzymatic antioxidants SOD and CAT, as well as the level of lipid peroxidation marker MDA and proinflammatory cytokines IL- $1 \beta$, IL- 6 and TNF- $\alpha$ were measured in accordance with the instructions of commercial kits. Values $(n=8)$ were presented as mean \pm standard deviation. D-gal, D-galactose; SOD, superoxide dismutase; CAT, catalase; MDA, malondialdehyde; IL, interleukin; TNF, tumor necrosis factor; Veh group, vehicle control group; Aging group, D-galinduced aging group. ${ }^{* *} \mathrm{p}<0.01$ compared to the Aging group. ${ }^{* \#} \mathrm{p}<0.01$ compared to the Veh group. 


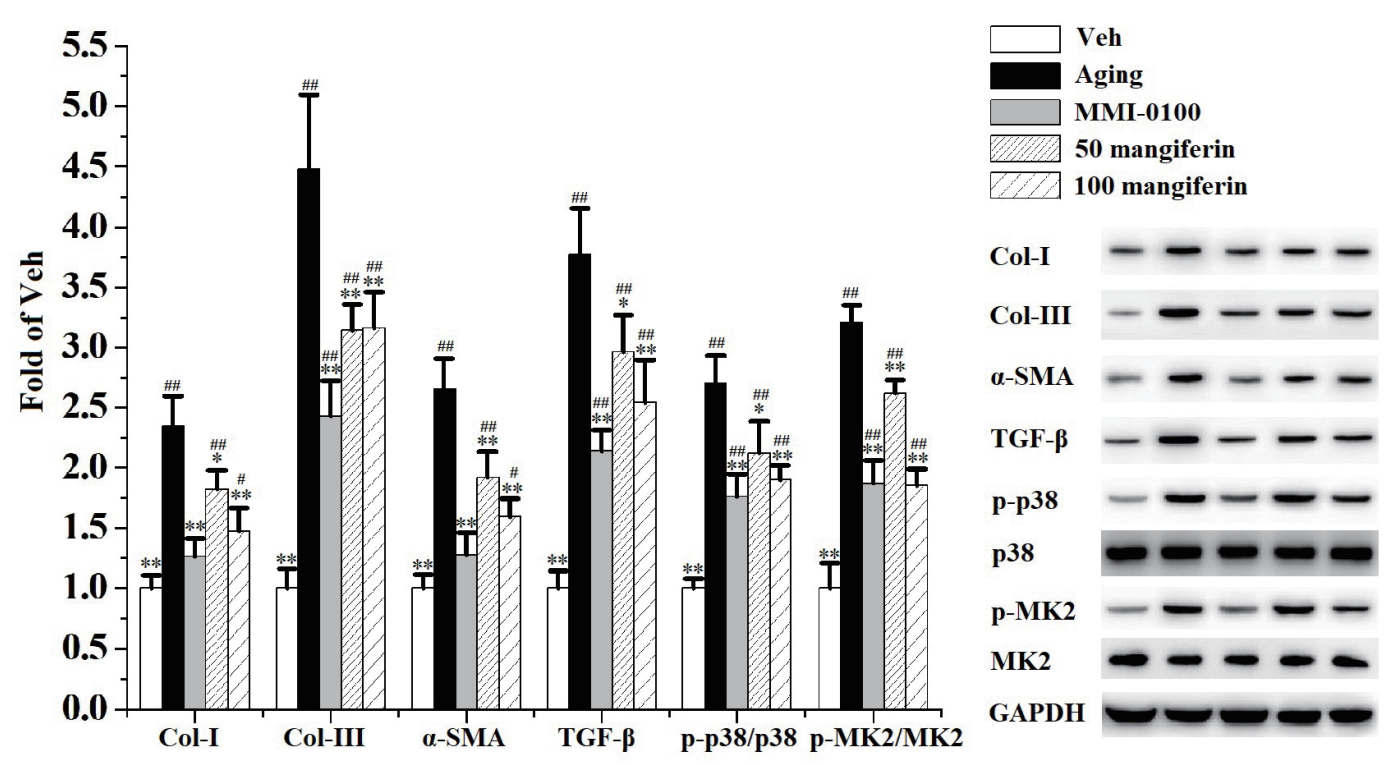

Fig. 4. Analysis for cardiac expression of pro-fibrogenic proteins (TGF- $\beta 1$, p-p38, p-MK2, p38, and MK2) and ECM proteins (Col-I, Col-III, and $\alpha$-SMA) in D-gal-induced aging rats. Values $(n=3)$ were presented as mean \pm standard deviation. D-gal, D-galactose; Veh group, vehicle control group; Aging group, D-gal-induced aging group. ${ }^{*} p<0.05,{ }^{* *} p<0.01$ compared to the Aging group. ${ }^{*} \mathrm{p}<0.05,{ }^{* \#} \mathrm{p}<0.01$ compared to the Veh group.

and $100 \mathrm{mg} / \mathrm{kg} / \mathrm{d}$ ) significantly increased the cardiac activities of SOD and CAT, decreased the cardiac levels of MDA, IL-1 $\beta$, IL- 6 and TNF- $\alpha$. It indicated that mangiferin attenuated D-galinduced cardiac oxidative stress and inflammation in rats.

\section{Mangiferin inhibited D-gal-induced cardiac pro- fibrogenic TGF- $\beta 1 /$ p38/MK2 signaling pathway}

Fig. 4 represents D-gal injection provokes cardiac pro-fibrogenic TGF- $\beta 1 /$ p38/MK2 signaling pathway in rats. When compared to the Veh, the cardiac expression of TGF- $\beta 1$, the phosphorylation levels of p38 and MK2, as well as the expression of ECM proteins Col-I, Col-III, and $\alpha$-SMA were significantly enhanced in rats from Aging group. When compared to the Aging group, MMI0100 and mangiferin (50 and $100 \mathrm{mg} / \mathrm{kg} / \mathrm{d}$ ) significantly reduced the cardiac expression of TGF- $\beta 1$, p-p38, p-MK2, Col-I, Col-III, and $\alpha$-SMA. The results showed that mangiferin inhibited $\mathrm{D}$ gal-induced cardiac pro-fibrogenic TGF- $\beta 1 / \mathrm{p} 38 / \mathrm{MK} 2$ signaling pathway.

\section{DISCUSSION}

Aging is the predisposing risk factor for cardiac hypertrophy, which severely harms the public health and brings huge time and financial burden [9]. D-gal has been long used to establish experimental aging models in animals because that it brings an abnormal D-gal accumulation in vivo and subsequently leads to harmful effects in the body [1]. The pathological progress of cardiac hypertrophy is accompanied by disorder expressed genes. ANP, $\mathrm{BNP}$, and $\beta$-MHC are suggested to be the most consistent mark- ers for cardiac hypertrophy $[3,17]$. Moreover, aging promotedcardiac fibrosis is the characteristic of age-related cardiac decline including cardiac hypertrophy $[4,8]$. Several studies demonstrated that mangiferin had the abilities of inhibiting pulmonary fibrosis [18], renal interstitial fibrosis [19,20] and cardiac fibrosis [13]. In our present study, mangiferin ameliorated D-gal-induced cardiac hypertrophy and fibrosis by reducing heart index, decreasing the serum levels of cardiac injury indicators and expression of hypertrophic makers, as well as inhibiting cardiac collagen deposition.

Aging is a complex physiological process. The oxidative stress is considered to be one of the possible mechanisms underlying aging-related pathological changes [9]. Evidences indicate that aging causes the decline in mitochondrial function and antioxidant defense systems in vivo [5,7]. Accumulated reactive oxygen species (ROS) leads to oxidative damage [21]. These ROS stimulate oxidative burst, and contribute to the damages of cell membrane and DNA [22]. As a major pathophysiological mechanism for heart diseases, oxidative stress participates in the occurrence and development of cardiac hypertrophy [7,21]. Under normal conditions, ROS are scavenged by cellular antioxidant defense mechanisms $[2,23]$. SOD and CAT are the core members of antioxidant enzymatic defense systems [10]. The activity of SOD is used as an indicator for aging since that SOD protects against ROS-induced cellular injury via diminishing toxic oxygen free radicals $[2,23]$. Similarly, CAT is responsible for catalyzing the harmful hydrogen peroxide into harmless water [2]. MDA is a secondary product of lipid peroxidation, which is triggered by oxygen free radicals [10]. It is found that the level of MDA increases within the mitochondria from failing hearts [23]. So, the content of MDA is used to evaluate the extent of cellular damage and widely used as the biomarker of oxidative stress $[2,10]$. Our study confirmed that 
mangiferin attenuated $\mathrm{D}$-gal-induced cardiac oxidative stress via enhancing the activities of SOD and CAT, as well as reducing the level of MDA.

Chronic inflammation is another pathological mechanism for CVDs besides oxidative stress [24]. The cardiac levels of proinflammatory mediators (like IL- 6 and TNF- $\alpha$ ) are over-generated under pathological stimuli [25]. D-gal elevates the levels of these inflammatory cytokines in heart [26]. Furthermore, local inflammation promotes myocyte hypertrophy and interstitial fibrosis, and finally induces cardiac hypertrophy and heart failure [25]. We found that mangiferin attenuated D-gal-induced cardiac inflammation via decreasing the cardiac levels of IL-1 $\beta$, IL- 6 , and TNF- $\alpha$.

Fibrosis is associated with ECM accumulation and collagen deposition, and subsequently leads to progressive architectural remodeling in nearly all organs [27]. Cardiac fibrosis is an important pathophysiological changes in cardiac aging and observed in several cardiac diseases [8,14]. Oxidative stress can up-regulate cardiac fibrotic signaling pathway $[8,17]$. For example, ROS can induce cardiac fibrosis via enhancing the expression of profibrotic TGF- $\beta 1$ [14]. The molecular mechanisms of organ fibrosis are not fully elucidated. In the process of cardiac fibrosis, fibroblasts are activated and transformed into myofibroblasts, and subsequently lead to ECM accumulation and collagen deposition [28]. TGF- $\beta 1$ is found to have the function of inducing cardiac myofibroblasts [29]. Emerging evidence suggests that profibrotic TGF- $\beta 1$ contains canonical and noncanonical signaling pathways in cardiac fibrosis [15]. On the one hand, TGF- $\beta 1 /$ Smad3 signaling pathway is considered to be the canonical fibrosis-regulated signaling pathway [30]. On the other hand, the noncanonical TGF- $\beta 1$ signaling pathway attracts more and more attentions.

MAPKs family proteins are involved in diverse cellular processes including cell differentiation, proliferation and apoptosis [31]. It is known that oxidative stress enhances the expression of MAPKs proteins [11]. P38 is a member of MAPKs. P38 signaling pathway can be activated by ROS [17]. The phosphorylation activation of $\mathrm{p} 38$ promotes the proliferation and differentiation of cardiac fibroblasts, as well as stimulates collagen deposition. Ultimately, cardiac fibrosis occur [32]. MK2 is a MAPK activated protein kinase belonging to serine-threonine kinase family. MK2 can be activated through phosphorylation under inflammation conditions [33]. MK2 is the downstream of p38. The phosphorylation level of MK2 is in direct proportion to the phosphorylation level of p38 [31]. MK2 is involved in regulating the levels of proinflammatory cytokines (such as IL-1 $\beta$, IL-6, and TNF- $\alpha$ ) [33]. Furthermore, MK2 participates in p38-induced heart failure [32]. As a result, MK2 seems to provide a bridge between oxidative stress and inflammation in cardiac fibrosis. Study illustrates that TGF- $\beta$ is the upstream of MK2 [11]. Blocking MK2 can inhibit TGF- $\beta$-induced fibroblast differentiation and ECM protein aSMA expression [15]. MK2 inhibitor MMI-0100 treatment successfully attenuates cardiac fibrosis and inflammation [32]. It can be concluded that the TGF- $\beta 1 / \mathrm{p} 38 / \mathrm{MK} 2$ is an important noncanonical fibrosis-regulated signaling pathway in age-related cardiac decline [30]. Previous study found that mangiferin alleviated cardiac fibrosis via activating Nrf2 signaling pathway, and thus promoting reduced glutathione (GSH) synthesis [13]. In our present work, we further identified the abilities of mangiferin on attenuating cardiac oxidative stress, inflammation and fibrosis via inhibiting the activation of TGF- $\beta 1 / \mathrm{p} 38 / \mathrm{MK} 2$ signaling pathway. MMI-0100 is a cell-penetrating peptide inhibitor of MK2 [34]. MMI-0100 had exerted anti-cardiac fibrosis ability [15]. So, MMI0100 was used as the positive control in this study. MMI-0100 and $100 \mathrm{mg} / \mathrm{kg} / \mathrm{d}$ mangiferin significantly attenuated age-related cardiac declines and fibrosis.

In conclusion, the whole findings suggested that mangiferin suppressed D-gal-induced cardiac aging, ameliorated cardiac oxidative stress, inflammation and fibrosis possibly via inhibiting TGF- $\beta / \mathrm{p} 38 / \mathrm{MK} 2$ signaling pathway. This present study provided new evidences for the protective effects of mangiferin against agerelated cardiac hypertrophy and fibrosis.

\section{ACKNOWLEDGEMENTS}

This research is supported by the Hubei Municipal Health Commission Scientific Research Project (grant numbers WJ2019H329) and by the Wuhan Municipal Health Commission Scientific Research Project (grant numbers WX19A14 and WZ15A01).

\section{CONFLICTS OF INTEREST}

The authors declare no conflicts of interest.

\section{REFERENCES}

1. Bo-Htay C, Palee S, Apaijai N, Chattipakorn SC, Chattipakorn N. Effects of D-galactose-induced ageing on the heart and its potential interventions. J Cell Mol Med. 2018;22:1392-1410.

2. Zhao X, Yi R, Zhou X, Mu J, Long X, Pan Y, Song JL, Park KY. Preventive effect of Lactobacillus plantarum KSFY02 isolated from naturally fermented yogurt from Xinjiang, China, on D-galactoseinduced oxidative aging in mice. J Dairy Sci. 2019;102:5899-5912.

3. Althurwi HN, Abdel-Kader MS, Alharthy KM, Salkini MA, Albaqami FF. Cymbopogon proximus essential oil protects rats against isoproterenol-induced cardiac hypertrophy and fibrosis. Molecules. 2020;25:1786.

4. Deng M, Yang S, Ji Y, Lu Y, Qiu M, Sheng Y, Sun W, Kong X. Overexpression of peptidase inhibitor 16 attenuates angiotensin II-induced cardiac fibrosis via regulating $\mathrm{HDAC1}$ of cardiac fibroblasts. $J$ Cell Mol Med. 2020;24:5249-5259.

5. Chen WK, Tsai YL, Shibu MA, Shen CY, Chang-Lee SN, Chen RJ, 
Yao CH, Ban B, Kuo WW, Huang CY. Exercise training augments Sirtl-signaling and attenuates cardiac inflammation in D-galactose induced-aging rats. Aging (Albany NY). 2018;10:4166-4174.

6. Dehghani A, Hafizibarjin Z, Najjari R, Kaseb F, Safari F. Resveratrol and 1,25-dihydroxyvitamin $\mathrm{D}$ co-administration protects the heart against D-galactose-induced aging in rats: evaluation of serum and cardiac levels of klotho. Aging Clin Exp Res. 2019;31:1195-1205.

7. Chang YM, Chang HH, Lin HJ, Tsai CC, Tsai CT, Chang HN, Lin SL, PadmaViswanadha V, Chen RJ, Huang CY. Inhibition of cardiac hypertrophy effects in D-galactose-induced senescent hearts by Alpinate Oxyphyllae Fructus treatment. Evid Based Complement Alternat Med. 2017;2017:2624384.

8. Murtha LA, Morten M, Schuliga MJ, Mabotuwana NS, Hardy SA, Waters DW, Burgess JK, Ngo DT, Sverdlov AL, Knight DA, Boyle AJ. The role of pathological aging in cardiac and pulmonary fibrosis. Aging Dis. 2019;10:419-428.

9. Zhu B, Zhang L, Liang C, Liu B, Pan X, Wang Y, Zhang Y, Zhang Y, Xie W, Yan B, Liu F, Yip HK, Yu XY, Li Y. Stem cell-derived exosomes prevent aging-induced cardiac dysfunction through a novel exosome/lncRNA MALAT1/NF- $\kappa \mathrm{B} / \mathrm{TNF}-\alpha$ signaling pathway. Oxid Med Cell Longev. 2019;2019:9739258.

10. Guan W, Liu Y, Liu Y, Wang Q, Ye HL, Cheng YG, Kuang HX, Jiang XC, Yang BY. Proteomics research on the protective effect of mangiferin on $\mathrm{H} 9 \mathrm{C} 2$ cell injury induced by $\mathrm{H}_{2} \mathrm{O}_{2}$. Molecules. 2019;24:1911.

11. Suchal K, Malik S, Gamad N, Malhotra RK, Goyal SN, Ojha S, Kumari S, Bhatia J, Arya DS. Mangiferin protect myocardial insults through modulation of MAPK/TGF- $\beta$ pathways. Eur J Pharmacol. 2016;776:34-43.

12. Jiang T, Han F, Gao G, Liu M. Mangiferin exert cardioprotective and anti-apoptotic effects in heart failure induced rats. Life Sci. 2020;249:117476.

13. Song J, Meng Y, Wang M, Li L, Liu Z, Zheng K, Wu L, Liu B, Hou F, Li A. Mangiferin activates Nrf2 to attenuate cardiac fibrosis via redistributing glutaminolysis-derived glutamate. Pharmacol Res. 2020;157:104845.

14. Chang YM, Tamilselvi S, Lin HJ, Tsai CC, Lin YM, Day CH, Viswanadha VP, Chang HN, Kuo WW, Huang CY. Alpinia oxyphylla Miq extract ameliorates cardiac fibrosis associated with D-galactose induced aging in rats. Environ Toxicol. 2019;34:172-178.

15. Meng Q, Bhandary B, Osinska H, James J, Xu N, Shay-Winkler K, Gulick J, Willis MS, Lander C, Robbins J. MMI-0100 inhibits cardiac fibrosis in a mouse model overexpressing cardiac myosin binding protein C. J Am Heart Assoc. 2017;6:e006590.

16. Li Q, Jiang W, Wan Z, Ni Y, Lei L, Wei J. Polyphyllin I attenuates pressure over-load induced cardiac hypertrophy via inhibition of Wnt/ß-catenin signaling pathway. Life Sci. 2020;252:117624.

17. Chen Y, Ge Z, Huang S, Zhou L, Zhai C, Chen Y, Hu Q, Cao W, Weng Y, Li Y. Delphinidin attenuates pathological cardiac hypertrophy via the AMPK/NOX/MAPK signaling pathway. Aging (Albany NY). 2020;12:5362-5383.

18. Jia L, Sun P, Gao H, Shen J, Gao Y, Meng C, Fu S, Yao H, Zhang G. Mangiferin attenuates bleomycin-induced pulmonary fibrosis in mice through inhibiting TLR4/p65 and TGF- $\beta 1 / \mathrm{Smad} 2 / 3$ pathway. J Pharm Pharmacol. 2019;71:1017-1028.

19. Song Y, Liu W, Tang K, Zang J, Li D, Gao H. Mangiferin alleviates renal interstitial fibrosis in streptozotocin-induced diabetic mice through regulating the PTEN/PI3K/Akt signaling pathway. J Diabetes Res. 2020;2020:9481720.

20. Li X, Yan Z, Carlström M, Tian J, Zhang X, Zhang W, Wu S, Ye F. Mangiferin ameliorates hyperuricemic nephropathy which is associated with downregulation of AQP2 and increased urinary uric acid excretion. Front Pharmacol. 2020;11:49.

21. Li C, Yan Q, Tang S, Xiao W, Tan Z. L-Theanine protects H9C2 cells from hydrogen peroxide-induced apoptosis by enhancing antioxidant capability. Med Sci Monit. 2018;24:2109-2118.

22. Al-Awaida W, Akash M, Aburubaiha Z, Talib WH, Shehadeh H. Chinese green tea consumption reduces oxidative stress, inflammation and tissues damage in smoke exposed rats. Iran J Basic Med Sci. 2014;17:740-746.

23. Liu J, Ai Y, Niu X, Shang F, Li Z, Liu H, Li W, Ma W, Chen R, Wei T, Li X, Li X. Taurine protects against cardiac dysfunction induced by pressure overload through SIRT1-p53 activation. Chem Biol Interact. 2020;317:108972.

24. Leong XY, Thanikachalam PV, Pandey M, Ramamurthy S. A systematic review of the protective role of swertiamarin in cardiac and metabolic diseases. Biomed Pharmacother. 2016;84:1051-1060.

25. Palomer X, Román-Azcona MS, Pizarro-Delgado J, Planavila A, Villarroya F, Valenzuela-Alcaraz B, Crispi F, Sepúlveda-Martínez Á, Miguel-Escalada I, Ferrer J, Nistal JF, García R, Davidson MM, Barroso E, Vázquez-Carrera M. SIRT3-mediated inhibition of FOS through histone $\mathrm{H} 3$ deacetylation prevents cardiac fibrosis and inflammation. Signal Transduct Target Ther. 2020;5:14.

26. El-Baz FK, Hussein RA, Saleh DO, Abdel Jaleel GAR. Zeaxanthin isolated from Dunaliella salina microalgae ameliorates age associated cardiac dysfunction in rats through stimulation of retinoid receptors. Mar Drugs. 2019;17:290.

27. Weiskirchen R, Weiskirchen S, Tacke F. Organ and tissue fibrosis: molecular signals, cellular mechanisms and translational implications. Mol Aspects Med. 2019;65:2-15.

28. Zhou B, Yu JW. A novel identified circular RNA, circRNA_010567, promotes myocardial fibrosis via suppressing miR-141 by targeting TGF- $\beta 1$. Biochem Biophys Res Commun. 2017;487:769-775.

29. Yang L, Wang B, Zhou Q, Wang Y, Liu X, Liu Z, Zhan Z. MicroRNA-21 prevents excessive inflammation and cardiac dysfunction after myocardial infarction through targeting KBTBD7. Cell Death Dis. 2018;9:769.

30. Tan S, Lu Y, Xu M, Huang X, Liu H, Jiang J, Wu B. $\beta$-Arrestin1 enhances liver fibrosis through autophagy-mediated Snail signaling. FASEB J. 2019;33:2000-2016.

31. Tanaka T, Iino M, Goto K. Sec6 enhances cell migration and suppresses apoptosis by elevating the phosphorylation of p38 MAPK, MK2, and HSP27. Cell Signal. 2018;49:1-16.

32. Xu L, Yates CC, Lockyer P, Xie L, Bevilacqua A, He J, Lander C, Patterson C, Willis M. MMI-0100 inhibits cardiac fibrosis in myocardial infarction by direct actions on cardiomyocytes and fibroblasts via MK2 inhibition. J Mol Cell Cardiol. 2014;77:86-101.

33. Singh RK, Najmi AK. Novel therapeutic potential of mitogenactivated protein kinase activated protein kinase 2 (MK2) in chronic airway inflammatory disorders. Curr Drug Targets. 2019;20:367379.

34. Wang Z, Liang XY, Chang X, Nie YY, Guo C, Jiang JH, Chang M. MMI-0100 ameliorates dextran sulfate sodium-induced colitis in mice through targeting MK2 pathway. Molecules. 2019;24:2832. 